\section{El desarrollo de las multialfabetizaciones en las experiencias de extensión}

Alicia W. de Camilloni

Profesora Emérita de la Universidad

de Buenos Aires, Argentina

Miembro Honorario de la Academia

Nacional de Educación.

acamilloni@arnet.com.ar
A 100 años de la Reforma Universitaria de 1918 /

Perspectivas

RECEPCIÓN: 07/09/17

ACEPTACIÓN FINAL: 24/09/17

\section{Resumen}

El escrito centra el análisis en el valor formativo que tienen cierto tipo de experiencias de extensión para los estudiantes universitarios. Es decir, en aquellas prácticas en la que los aprendizajes académicos y profesionales se ponen a prueba en situaciones auténticas, en las que los estudiantes se comprometen en una relación responsable con la comunidad.

Para dar cuenta de este valor formativo, se recurre al concepto de "multialfabetización" como una noción útil que posibilita dar cuenta de la heterogeneidad, multiplicidad, complejidad, dinamismo e integralidad de los aprendizajes que se ponen en juego en estas situaciones pedagógicas.

En este sentido, las prácticas de extensión de educación experiencial ofrecen, señaladamente, situaciones de participación en las que las habilidades requeridas por la multialfabetización encuentran un cauce propicio porque su desarrollo se plantea como una necesidad en el trabajo con problemas auténticos.

Palabras clave

- Prácticas de extensión

- Educación experiencial

- Multialfabetización

\section{Resumo}

A matéria foca a análise no valor fomativo que têm determinado tipo de esperiências de extensão para os estudantes universitários. Quer dizer, naquelas práticas na que as aprendizagens acadêmicas e profissionais são testadas em situações autênticas, nas que os estudante se comprometem em uma relação responsável com a comunidade.

Para informar sobre este valor formativo se recorre ao conceito de "multialfabetização" como uma noção útil que possibilita dar conta da heterogeneidade, multiplicidade, complexidade, dinamismo e integridade das aprendizagens que estão em jogo nestas situações pedagógicas. Neste sentido, as práticas de extensão de educação experiencial oferecem, claramente, situações de participação em que as habilidades requeridas pela multialfabetização encontram um caminho propício porque seu desenvolvimento se apresenta como uma necessidade no trabalho com problemas autênticos.

Palavras-chave

- Práticas de estensão

- Educação experiencial

- Multialfabetização
El presente escrito fue preparado por la profesora Camilloni para la Conferencia central del III Congreso de Extensión de AUGM "Democracia, derechos humanos e inclusión social. Camino a los 100 años de la Reforma Universitaria" realizada en la Universidad Nacional del Litoral el 7 de septiembre de 2017. El texto fue cedido por la autora y el trabajo de edición estuvo a cargo de Cecilia lucci.
Para citación de este artículo Camilloni, A. (2017). El desarrollo de las multialfabetizaciones en las experiencias de extensión. Revista +E versión en línea, 7(7), 60-67. Santa Fe, Argentina: Ediciones UNL. 
¿Qué valor formativo tienen para los estudiantes las experiencias de participación en proyectos de extensión?

La participación de los estudiantes en prácticas de extensión habilita aprendizajes que tienen características diversas de las que presentan los que realizan en las actividades convencionales, particularmente porque, en atención a su carácter propio, se desarrollan en situaciones auténticas, esto es, de la vida real, en las que se comprometen en una relación responsable con la comunidad. Los aprendizajes académicos y los aprendizajes profesionales se ponen a prueba en la acción. Su multiplicidad es importante, en lo que se refiere tanto a número y heterogeneidad como a relevancia. Efectivamente, estos aprendizajes importan. La formación que reciben los estudiantes se puede analizar desde distintas perspectivas. La vincularé acá con el concepto de "multialfabetizaciones", noción útil, en este caso, porque da cuenta, a un tiempo, de la variedad, multiplicidad, complejidad, dinamismo e integralidad de los aprendizajes que se ponen en juego en estas situaciones pedagógicas.

El concepto de "alfabetización" está asociado a la enseñanza de la escritura. Alfabetizar es dar a conocer los signos que se emplean para escribir: de alfa a beta y así de seguido, hasta omega. En el siglo XX adquiere un sentido metafórico. Se multiplican las alfabetizaciones. Pero no es esta la consecuencia de sucesivas fragmentaciones del concepto original, no es la simple resultante de un agregado técnico de códigos. En un profundo cambio cultural, nuevos códigos, no siempre articulados entre sí, generan y son generados por una extraordinaria ampliación del horizonte de la vida y, en consecuencia, de nuevos significados para la humanidad. Si la lengua, citando a Roland Barthes (1967:18), funciona "como una negatividad, el límite inicial de lo posible", cada uno de estos códigos se instala como un nuevo límite inicial de posibilidad. ¿Son realmente códigos nuevos? Algunos existen desde hace siglos, otros son funcionalmente nuevos. Pero al alcanzar un nuevo estatus a través del reconocimiento institucional, se curricularizan y legitiman como nuevas modalidades de la alfabetización.

Alcanzan un nuevo destino social. Se enriquece, de este modo, el concepto de escritura. Formas de expresión nuevas y antiguas no se limitan a la complementación respecto de la alfabetización en los signos y sus correspondientes sonidos. La revolución escritural alcanza a los significados de un modo que ya había sido anunciado por McLuhan. El mensaje estalla. Códigos y convenciones se transforman y se rompen ante los ojos mismos de quienes tratan de preservarlos para que no resulten devorados por el tiempo presente. La inclusión en el currículo de nuevos códigos es una batalla por las ideas, las palabras y las relaciones sociales. Implanta identidades personales, académicas y profesionales en procesos de socialización fuertemente guiados por la autoridad académica. En respuesta al estallido de los significados del concepto metafórico de alfabetización, su codificación y estandarización en la práctica suponen más bien que la liberación, la búsqueda de un encuadramiento del pensamiento. Este se ha ampliado hacia nuevas formas de expresión. Es preciso contenerlas y formarlas. Colocarlas en el redil. Mostrarles los caminos adecuados y apropiárselas antes de que se escapen hacia un mundo en el que la cultura legítima ya no pueda alcanzarlas, en un tiempo que ya no es ni único ni homogéneo.

Las Ilamadas alfabetizaciones académicas se centran, especialmente, en aspectos disciplinarios y comunicacionales y tienen como propósito el desarrollo de capacidades de habla, escucha, escritura, lectura y cálculo mediante el empleo de diversos códigos. Se las reconoce como prácticas sociales históricas y se las liga a los contextos en los que se despliegan. Están asociadas, principalmente, a aprendizajes verbales, icónicos y de cálculo, a diferentes formas de discursos y textos, incluidos los lenguajes digitales, en un sentido amplio, en el marco de lo que llana Snyder denomina "nuevo orden comunicacional" (2001). Se establecen como vías de aprendizaje fuertes relaciones con ciertos tipos de trabajo intelectual asociados a la conformación de sólidas estructuras de conocimiento. Las alfabetizaciones son, ciertamente, múltiples. Se enumeran diferentes clases que son categorizadas según distintas claves: el tipo de código, el tipo de disciplina, el tipo de género discursivo, por ejemplo. Cada una de ellas se divide en subalfabetizaciones según la especialización en la que las estructuras se configuran. En su Diccionario de alfabetización, de 1995, Harris y Hodge listan 38 alfabetizaciones distintas. La educación experiencial está basada en la interdisciplinariedad y el aprendizaje constructivista. Las prácticas habituales en la alfabetización académica, en cambio, no suelen destinarse a la generación de aprendizajes interdisciplinarios. En este sentido, nos interesa realzar la riqueza y variedad de los aprendizajes que los estudiantes tienen la oportunidad de desarrollar en sus experiencias de participación en actividades de extensión y que poseen un valor formativo insustituible desde una perspectiva pedagógica que pretende dar cuenta de las condiciones y exigencias que los desempeños personales, sociales, culturales y profesionales imponen actualmente a la enseñanza universitaria.

La preocupación por la necesidad de satisfacer esta misión de formación por las universidades y la enseñanza superior en general se viene manifestando progresivamente y orienta un trabajo de definición de aprendizajes en diferentes dominios desarrollado en el marco amplio del concepto de educación experiencial de David Kolb (1984), que ha servido de base para la categorización de clases de actividades de extensión en algunos modelos en uso en programas destinados a formalizar la integración en el currículo de estas actividades. Con el propósito de ofrecer elementos conceptuales que faciliten la programación, implementación y evaluación de la vinculación entre las misiones de docencia, investigación y extensión de las universidades, procuraremos examinar críticamente 
66

Reemplazar el concepto de alfabetización

por el de multialfabetizaciones, como

noción nueva, tiene el objetivo de

superar las limitaciones de los enfoques

tradicionales algunas ideas atinentes a estas cuestiones, centrándonos en los aspectos pedagógicos que están implicados en ellas. Como señalamos, la noción de multialfabetización nos acompañará en el análisis de los conceptos de experiencia, educación experiencial, aprendizajes y contenidos de la formación.

\section{¿Por qué hablamos de multialfabetización?}

La historia de su postulación conceptual es particularmente interesante y, a nuestro juicio, presenta un desarrollo con rasgos analógicos al del concepto de educación experiencial. La alfabetización se definió inicialmente como el eje central de la educación escolar. En una cultura de signos, el aprendizaje del manejo y comprensión de los signos era condición de ciudadanía. Leer y comprender, escribir y emplear letras y números eran saberes indispensables para la participación en la vida urbana y, a la vez, eran una puerta para la adquisición de nuevos conocimientos. Enseñar estos saberes significaba enseñar por signos (en-seigner), enseñar a de-signar, a denominar por signos. La alfabetización estuvo centrada en el dominio de la lectura y la escritura. Los niveles de definición de la alfabetización fueron evolucionando históricamente y con ellos los requerimientos de formación. Las tasas de alfabetización se convirtieron en uno de los indicadores de desarrollo socioeconómico de los países. En los censos de población, sin embargo, la pregunta se limitaba a recoger información por terceras personas acerca de si cada habitante decía saber leer y escribir. La definición escolar progresó en el sentido de requerir demostrar esa capacidad, pero la capacidad misma fue recibiendo exigencias mayores: comprender el significado de un texto y producir un texto con significado; comprender y construir significados en un determinado contexto cultural; construir el significado de un texto interactuando con este; demostrar dominio del lenguaje escrito relacionado con el pensar; dominar el lenguaje escrito y oral y el cálculo relacionado con el pensar; dominar el lenguaje escrito y oral, el cálculo y la escucha relacionados con el pensar. Características de estas definiciones que se acuñaron en la cultura escolar que se sustentaba sobre un concepto unitario de alfabetización, restringido, formalizado, monolingüe, monocultural y gobernado por las reglas del lenguaje. Pero surgió un reclamo en el sentido de señalar la necesidad de pensar en los nuevos requerimientos que era imprescindible satisfacer, se acuñó la expresión "analfabeto funcional” y, en consecuencia, la de "alfabetización funcional". En una publicación de 1956 de la UNESCO, William S. Gray (1956:21), respecto de la educación de adultos, retomó la idea de una alfabetización funcional al afirmar que supone la "capacidad para leer y escribir independientemente de acuerdo con las demandas que se reciben". La expresión "alfabetización funcional" se fue ampliando y se la refiere hoy a la adquisición de habilidades verbales, cognitivas y computacionales apropiadas para cumplimentar fines prácticos en situaciones culturales específicas. Así, por ejemplo, la OCDE, en estadísticas de Canadá, define a la alfabetización "como la capacidad para comprender y usar información escrita en la vida cotidiana, en el hogar, en el trabajo y en la vida social con el fin de lograr objetivos personales y ampliar el conocimiento y las capacidades propias". (1995)

La conceptualización de la alfabetización responde, pues, a condiciones históricas. Los estudios sobre la alfabetización son interdisciplinarios y la pedagogía, la psicología, la historia, la lingüística y la antropología social aportan a su definición y a las modalidades de su enseñanza. Los estudios ya no son solo cuantitativos sino que son cuanti y cualitativos. Surgen alfabetizaciones múltiples. Gerard Fourez (1997:907-936) afirma que "en nuestras sociedades hay ciertos saberes y haceres que se han vuelto tan útiles para los jóvenes como la lectura, la escritura y el cálculo". A ellos se refiere, por ejemplo, Douglas Kellner (2004; Kant, Kellner, 2006) como "las alfabetizaciones postipográficas" (audiovisual, en medios, digital), a las que se añadieron las alfabetizaciones disciplinarias (científica [Hurd, 1958:13-16; McCurdy, 1958:366-368] tecnológica) y otras más generales como la alfabetización informacional. Se amplió, enriqueció y diferenció internamente el concepto de comunicación. La metáfora, estrechamente ligada al empleo de símbolos gráficos, se diversificó en su uso, y lo que para algunos se había convertido en un sinónimo de educación o, al menos, de educación escolar, ligada al dominio de conocimientos básicos, evolucionó hacia 
la conformación de una panoplia de saberes diferenciados, con límites propios, especialistas y especialidades, y niveles crecientes de profundidad exigida. El concepto de alfabetización unitario sufrió una explosión. No hay alfabetización sino alfabetizaciones, diversificadas y especializadas. Por lo tanto, lo que antes pretendía ser una unidad se quebró en las últimas décadas del siglo pasado. La limitación de campos de conocimiento creó condiciones para su enriquecimiento en profundidad, pero a costa no solamente de su estrechamiento sino de su aislamiento mutuo.

Un movimiento que se propuso examinar críticamente la construcción del campo fue iniciado en 1994 por un grupo de diez especialistas en temas de alfabetización y comunicación de Estados Unidos, Reino Unido y Australia. El grupo, que adoptó el nombre de New London Group, reunido en New Hampshire, acuñó un nuevo término: "multialfabetizaciones". Sus ideas se plasmaron en el trabajo "Una pedagogía de las multialfabetizaciones:

el diseño de futuros sociales" (1996), que fue considerado el Manifiesto del movimiento.

Este grupo estudia las conexiones entre el cambiante medio social y los profesores y estudiantes. A partir de la crítica que realiza a la configuración del campo de la alfabetización de su tiempo, propone un nuevo enfoque de la pedagogía de alfabetización que denomina "multialfabetizaciones". Y afirma que la multiplicidad de canales de comunicación y la creciente diversidad cultural y lingüística en el mundo actual convocan a adoptar una visión mucho más amplia de la alfabetización que la tradicional. Se proponen "intentar ampliar el entendimiento de la alfabetización y de la enseñanza y el aprendizaje de la alfabetización" (New London Group:61) para incluir la multiplicidad de lenguajes y formas textuales. El término alude a la gran variedad de multiplicidades entendida ahora como textos válidos, las prácticas de alfabetización y las relaciones semióticas. Reemplazar el concepto de alfabetización por el de multialfabetizaciones, como noción nueva, tiene el objetivo de superar las limitaciones de los enfoques tradicionales al señalar que la intensificación de las negociaciones entre las múltiples diferencias lingüísticas y culturales es central para la práctica de la vida laboral, cívica y privada de los estudiantes. Estos logros estimularían el compromiso crítico necesario para diseñar sus futuros sociales y tener éxito en las metas que se propusieran alcanzar. El New London Grup se posicionó en la necesidad de desarrollar una enseñanza apoyada en proyectos que consideren que la diversidad debe hacerse visible en las dimensiones sociales, personales y de ciudadanía. Como concepto clave para trabajar las dimensiones de la vida social, proponen la noción de diseño, relacionada con el mundo del trabajo y la profesión, con la construcción de la identidad y con la participación ciudadana, y señalan la importancia de la labor de interpretaciones y resignificaciones que surgen en diferentes contextos, la cual las hace susceptibles de cambios y transformaciones. Ante las preguntas que se formulan acerca de cómo operacionalizar sus ideas, su respuesta se orienta a recomendar el uso de una pedagogía práctica situada en el mundo real, con enseñanza intencional explícita, con un enfoque crítico y de práctica transformadora.

En 2009, 15 años después de la iniciación del movimiento, dos de los integrantes del grupo, Cope y Kalantzis, efectuaron un balance de lo producido sobre la base de sus ideas y propuestas. Su conclusión fue la siguiente:

"Hemos encontrado que la forma básica de nuestra posición original ha superado la prueba del tiempo. De hecho, resulta una guía útil para la comprensión y la práctica, la importancia de la diversidad, la noción de diseño como construcción activa del significado, la importancia de la multimodalidad y la necesidad de un enfoque más holístico en la pedagogía”. (164-195)

Actualmente, las diversas alfabetizaciones se estudian en su relación con las prácticas sociales: el uso del lenguaje presenta variabilidad en la construcción de significados en diferentes contextos sociales, culturales y profesionales. Y se parte del principio de que los usos son diferentes en distintos contextos. No hay un único concepto que se corresponda con una alfabetización ideal. Es un concepto que implica su heterogeneidad y pluralidad interna: variedad, rupturas, contradicciones, inacabamiento, cambio, crecimiento, regresiones, movimientos pendulares. Hay alfabetizaciones emergentes. Múltiples discursos. Múltiples voces y múltiples oyentes. A diferencia de este, el cuadro general en el que se inscriben las multialfabetizaciones ha sido 
caracterizado por distintos autores. N. Burbules (2009) lo denomina como de aprendizaje "ubicuo" tanto en lo que se refiere a la ubicación temporal como a la ubicación espacial. Nuevos entornos de aprendizaje formales y no formales son los escenarios en los que ocurren los aprendizajes, con empleo, como lo señalara Basil Bernstein (1975), de pedagogías visibles e invisibles. Se señala la permeabilidad de la escuela en su relación con la sociedad. Se instalan propuestas de entornos personales de aprendizaje como enfoque pedagógico de aprendizajes formales e informales con uso de redes sociales para apoyar aprendizajes autorregulados en contextos de educación superior. Y, como afirman Tyner, Gutiérrez Martín y Torrego González, "en la raíz de todos estos cambios derivados de la convergencia de medios, lenguajes, sistemas, métodos y entornos, está la digitalización". (2015:45)

El amplificado concepto de multialfabetización se concentra crítica y profundamente en las dimensiones multilinguales y multimodales de la comunicación. Estos autores se refieren a la educomunicación, a la que caracterizan como universal, continua, "glocal", significativa, crítica y creativa, conjunta para la escuela, los medios y el entorno cercano, colaborativa y participativa, flexible y abierta a las innovaciones continuas, holística, globalizada y transdisciplinar, y equilibrada e integradora de la teoría y la práctica, asociada con la reflexión-acción, abierta al aprendizaje individual y social, presencial y virtual e intercultural. Los autores concluyen que la multialfabetización que proponen

"para la cultura de la participación en la era de la convergencia

(...) solo puede darse si sabemos sacar lo mejor de todos y cada uno de los entornos de aprendizaje reales y virtuales que constituyen el continuum de nuestra vida". (2015: 54)

Cuando examinamos la historia del concepto de multialfabetización partiendo de las definiciones sucesivas de la alfabetización, reconocemos dos etapas claramente demarcadas. Por una parte, en el transcurso del siglo XX se pasa de una definición unitaria a un proceso en el que la alfabetización estalla en multitud de alfabetizaciones específicas que se diversifican y especializan, son generadas y generan multitud de comunidades académicas que investigan, difunden sus ideas y enseñan. Por otra parte, a finales del siglo, en una segunda etapa, la idea de multialfabetización, igualmente con el aporte de muchos autores, responde a un proyecto opuesto que, sin ignorar la importancia de los desarrollos teóricos y prácticos de las especializaciones, procura recuperar la integralidad del significado de la comunicación humana y brindarle las condiciones para construir significados en términos de prácticas sociales generales, aunque situadas, con la aspiración de que sean transferibles a otras presentes y futuras situaciones. No se trata solamente de desarrollar habilidades técnicas en el uso y manejo de medios o de conocimientos disciplinares, sino de reflexionar, comprender, diseñar, crear, trascender las situaciones presentes y ser capaz de imaginar futuros posibles.
Si el proceso seguido en el caso de la alfabetización parece tener el carácter de explosión, cuando la alfabetización trata de recuperar su sentido integral, su significado da lugar a una nueva construcción tan profunda que asume el formato de una implosión. No se trata de manejo de códigos, de uso de signos para la comunicación sino de la apertura a la arquitectura de nuevos significados que atañen a personas, sociedades y culturas que se comunican e interactúan entre sí con propósitos definidos en un gran horizonte de apertura cultural.

\section{¿Por qué son importantes las prácticas de extensión de educación experiencial?}

El concepto de educación experiencial reúne características semejantes a las que hemos hallado en el de multialfabetizaciones. Su historia también se edifica sobre principios pedagógicos de ubicuidad, continuidad, convergencia y permeabilidad. $Y$ es, igualmente, el resultado de un proceso en el que se fue profundizando progresivamente el significado del concepto de aprendizaje por experiencia ampliándose en el sentido de una indagación dirigida hacia el interior de su aporte existencial a la formación de los estudiantes. Veremos, entonces, que los dos conceptos se enriquecen mutuamente.

Cuando la educación experiencial se incorpora a la educación formal debe salvar tres peligros que podrían desnaturalizarla. El primero, la confusión con el "aprender haciendo"; el segundo, el limitarse al uso de una metodología de la enseñanza de aprendizaje en ciclos; el tercero, el encierro en un único campo disciplinario. En el primer caso, la educación experiencial se diferencia del "aprender haciendo", en que la reflexión del estudiante sobre el hacer es la fuente de su aprendizaje. Conoce teoría, se prepara para la acción y cuando actúa piensa de un modo accional. Y así lo describen Hampson y Norris cuando sostienen que "la cognición, en general, y la acción, en particular, no son operaciones arbitrarias, son actividades llevadas a cabo por una persona con necesidades reales y emociones reales" (1996:182).

Jerome Bruner, en Actos de significado (1991) coloca como atributos de un pensamiento ligado a la acción que, dado que su desarrollo acontece en diferentes contextos sociales que favorecen su producción, es promovido por las condiciones situacionales y es impulsado por la selección entre alternativas. De esta manera, se comprende que la experiencia personal articula con el conocimiento teórico en el proceso de deliberación siempre que existan alternativas de acción, libertad para sopesarlas y capacidad para tomar decisiones. Perkins y Salomon (1989:16-25) señalaron que esto ocurre cuando se llama la atención del aprendiz sobre las conexiones que se pueden hallar entre la teoría y la práctica y se lo impulsa para que las examine. La reflexión es demandante y, por lo general, los estudiantes no han recibido capacitación para profundizar en sus fundamentos y supuestos y para preparar 


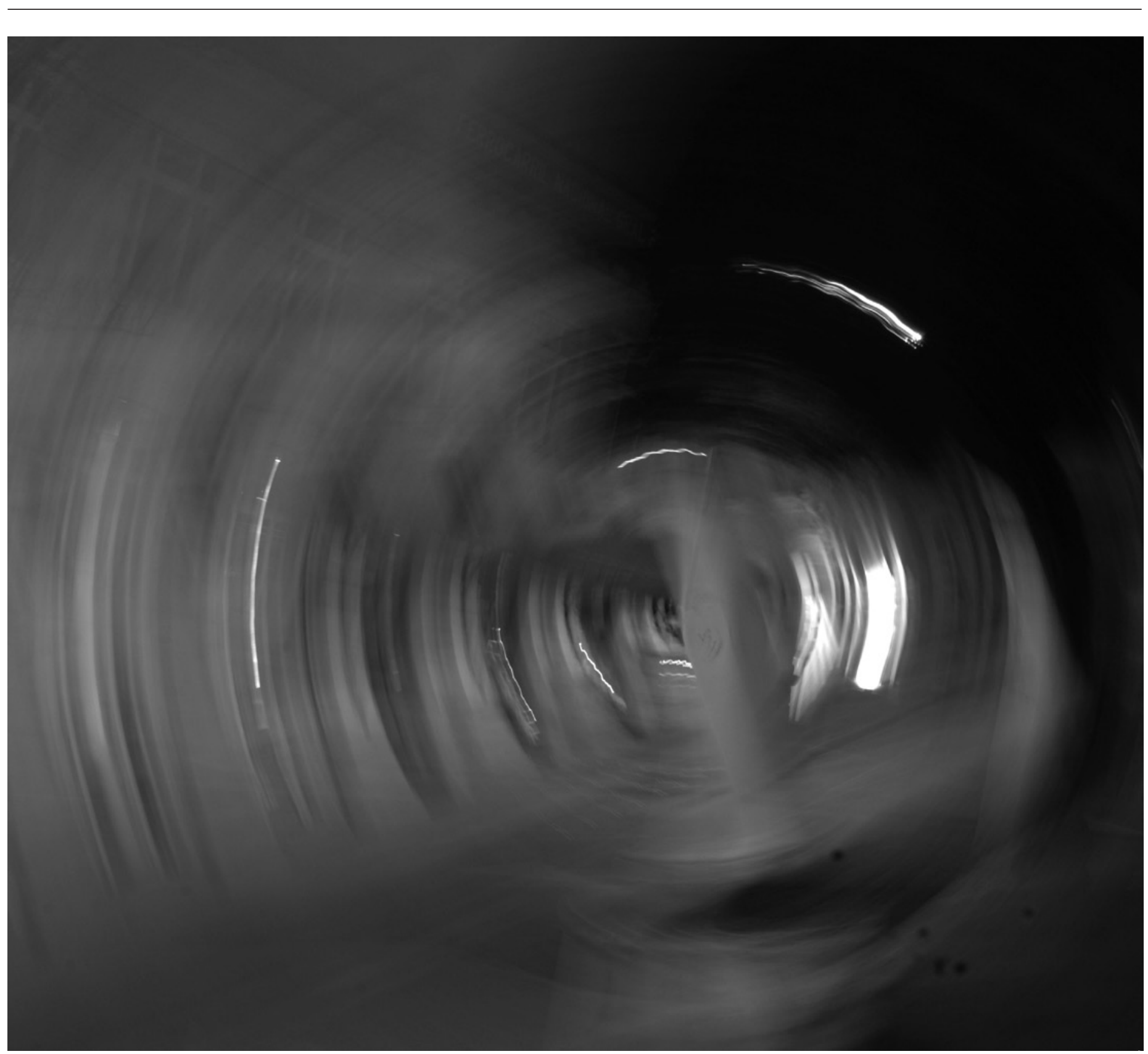

() Hugo Pascucci

\section{6}

Cuando la educación experiencial se incorpora a la educación formal debe salvar tres peligros que podrían desnaturalizarla. El primero, la confusión con el "aprender haciendo"; el segundo, el limitarse al uso de una metodología de la enseñanza de aprendizaje en ciclos; el tercero, el encierro en un único campo disciplinario 
y analizar sus argumentaciones e inferencias. La reflexión, por lo tanto, debe ser guiada y estimulada y, como afirman Sullivan y Rosin, debe ser "intensa"(2008). Los estudiantes deben tener conciencia de que esas experiencias son oportunidades valiosas de aprendizaje y no solo ocasiones para a aplicar lo que ya sabían. Las experiencias siempre son solo potencialmente educativas. Mal conducidas o mal trabajadas o fuera de la órbita de los intereses del estudiante, como había observado hace mucho tiempo John Dewey, pueden ser antieducativas. Para que esto no ocurra, se requiere que todos los involucrados participen en el decurso de la reflexión, que puede ser individual, grupal o colectiva, implicando a estudiantes, docentes y socios. La reflexión requiere tiempo y dedicación. En principio, es personalística, pero las condiciones de intersubjetividad en las que estas situaciones de aprendizaje se ubican contribuye a que su configuración resulte de un debate de ideas y valores y que sirva como recurso para el aprendizaje de la reflexión y de sus múltiples expresiones. Por esta razón, un aspecto interesante de este recorrido es la elección de una forma de expresión de la reflexión, de su marcha y de sus conclusiones, que no tienen por qué ser necesariamente consensuadas entre todos los participantes. Las diferencias se respetan y se incentivan, no simplemente se toleran. La diversidad entre personas y entre ideas es una de las riquezas que estas experiencias poseen. Individual o compartido, el registro facilita la construcción de nuevas estructuras de conocimiento. De acuerdo con Jack Mezirow (1997), se trata de una reflexión que es semejante a la resolución de problemas, pero ella no supone, únicamente, poner en cuestión la solución dada a una situación problemática externa sino iluminar al sujeto reflexionante mismo, sus conocimientos, ideas y acciones, esto es, a un sujeto que analiza el porqué, el qué y el cómo de sus ideas y acciones con el propósito de revisarlas y transformarlas si ello resultara necesario. De ahí que Mezirow afirme:

"But in contemporary societies we must learn to make our own interpretations rather than act on the purposes, beliefs, judgments, and feelings of others. Facilitating such understanding is the cardinal goal of adult education. Transformative learning develops autonomous thinking". (1997:5) ${ }^{1}$

Un segundo peligro que acecha a la educación experiencial es que las cuatro fases del ciclo de aprendizaje de David Kolb se conviertan en el plan ideal de la enseñanza y que se encierren en una propuesta de metodología de la enseñanza, al modo de un algoritmo que brinda una solución siempre exitosa. Las fases del ciclo representan un modelo muy interesante y muy valorado en el campo pedagógico. Se lo reconoce como estrategia de enseñanza y como método para el trabajo de campo. Pero debe ser interpretado siempre como componente de un continuo espiralado en el que, a partir de una situación específica en la que se realiza una experiencia concreta, se observa reflexivamente la situación y luego se conceptualiza el problema y se genera una nueva acción con significado sobre la situación, lo cual da lugar a una nueva experiencia concreta, y así de seguido. La reflexión a la que antes nos referimos es sintetizadora del aprendizaje seriado en ciclos sucesivos. En ella importa, fundamentalmente, la autoevaluación que hace el estudiante en dos planos: el que se refiere a aquello que él mismo ha aprendido y la contribución que ha efectuado para el logro de los objetivos del proyecto en el que ha participado. El tercer peligro al que antes aludimos es el de adoptar, para definir los aprendizajes y las acciones de los estudiantes, un esquema diferenciado y enclaustrado disciplinariamente al modo de una grilla de posicionamientos diversificados según tema o disciplina o profesión futura de los estudiantes miembros del grupo que lleva a cabo el proyecto. En las experiencias de participación en trabajos de extensión, las perspectivas monodisciplinares o monoprofesionales, que no explotan la riqueza propia de esas situaciones, constituyen un verdadero derroche de oportunidades de aprendizaje que no se pueden sustituir por otra clase de experiencias. Interesa aquí apelar a los criterios que hemos visto que se han propuesto para caracterizar a las multialfabetizaciones. Aunque no restringidas estas al campo de la comunicación, sino ampliadas a todas las formas de acción en las prácticas sociales, personales y profesionales, las características que mencionamos encuentran un lugar muy propicio para desarrollarse en estas experiencias de participación en proyectos de extensión.

Por estos peculiares rasgos, ponemos la atención en conocimientos y habilidades aprendidas y transformadas en la interacción de la teoría y la práctica. Dos tipos se suelen diferenciar: las habilidades cognitivas y las habilidades no cognitivas. Las cognitivas, viejas conocidas de los académicos profesores e investigadores, son objeto habitual de la enseñanza programada sistemáticamente. Las modalidades de evaluación en uso están fuertemente estandarizadas y señalan a los estudiantes la importancia de su formación. Pero no ocurre lo mismo con las habilidades no cognitivas. Estas se desarrollan en la educación, simultáneamente con las habilidades cognitivas, pero no de la misma manera. Se han enseñado siempre pero sin ser explicitadas, sin otorgar tiempo especial para su desenvolvimiento; cuando hay alguna programación, esta no reviste sistematicidad. Además, no suelen ser objeto de evaluación sino en casos extremos y, por lo general, están asociadas a sanciones y raramente a recompensas. En las experiencias de participación en proyectos de extensión, por el contrario, las habilidades no cognitivas, siempre articuladas
1) "En las sociedades contemporáneas debemos aprender a hacer nuestras propias interpretaciones en lugar de actuar de acuerdo con los propósitos, creencias, juicios y sentimientos de otros. Facilitar esta comprensión es el objetivo principal del aprendizaje transformativo, la conformación de un pensamiento autónomo" (aclaración de la autora). 
con las cognitivas, tienen una posición muy relevante. Estos aprendizajes son objeto de una pedagogía visible que los incluye expresamente.

Cuando nos preguntamos cuál es el valor formativo de la participación de los estudiantes en trabajos de extensión es menester poner especial cuidado en el desarrollo de las habilidades no cognitivas. Estas han sido objeto de variados estudios, en particular, en circunstancias y con públicos propios de la educación no formal. Sin embargo, forman parte de todos los enunciados de perfiles académicos y profesionales. Han sido objeto de distintas categorizaciones de las que tomaremos una que, por su aparente sencillez, nos permite sintetizar sus rasgos distintivos. Kai Zhou (2016) propone, en razón de su importancia, sus alcances y la posibilidad de ser enseñadas y aprendidas, que se acentúe la concentración en el desarrollo de tres habilidades no-cognitivas: la perseverancia y la fortaleza, el autocontrol y las habilidades sociales. De cada una hace una caracterización para fundamentar su elección.

Perseverancia y fortaleza se definen por la consistencia del interés y la persistencia en el esfuerzo. El autocontrol se define como "la capacidad de modificar las repuestas propias para alinearlas con ideales, valores, y aspiraciones sociales con vistas a logros de largo plazo". Se lo asocia a la autorregulación, a la autodisciplina, a la fuerza de voluntad y, como hemos visto, a la aceptación de recompensas diferidas. Las habilidades sociales son definidas de manera general como las habilidades para mantener relaciones efectivas y compatibles con otras personas. Un aspecto de orden cultural importante en situaciones de interculturalidad es la expresión de emociones. Estas tienen un importante papel en las situaciones de aprendizaje en relación con personas y grupos desconocidos, subculturas diferentes, espacios desconocidos, situaciones inesperadas.

Si bien se piensa que las habilidades cognitivas deben desarrollarse en las situaciones convencionales de enseñanza universitaria, su puesta en relación con situaciones vividas en la realidad las pone a prueba y esto se proyecta sobre la fortaleza de ideas y el carácter de los estudiantes que, por primera vez, afrontan situaciones problemáticas en las que tienen que tomar decisiones de la manera en que deberán hacerlo en su futuro desempeño profesional. La educación experiencial tiene, así, un fuerte efecto de retorno sobre el trabajo y el estudio en las aulas y en los modos formales tradicionales de enseñanza. Ambos interactúan y deben articularse. La extensión, por ende, enriquece asimismo su significado por este camino. No se limita a ofrecer conocimiento y acción al exterior en cumplimiento de una misión social que le permite, a la vez, enseñar a sus socios comunitarios y aprender de y con ellos. Su valor se extiende al interior más profundo de la propia universidad. A las vivencias y a la construcción de las identidades de sus estudiantes. $\mathrm{Y}$, en la medida en que se trate de experiencias compartidas, se extiende, igualmente, al mundo interior de sus docentes.

\section{Referencias bibliográficas}

Bernstein, B. (1975). Class Codes and Control. Vol. 3 Towards a Theory of Educational Transmissions. London: R.K.

Barthes, R. (1967). El grado 0 de la escritura. Buenos Aires: Jorge Álvarez.

Cope, B.; Kalantzis, M. (2009). "Multiliteracies": New Literacies, New Learning.

Pedagogies: An International Journal, 4(3), 164-195.

Bruner, J. (1991). Actos de significado. Más allá de la revolución cognitiva. Madrid: Alianza.

Burbules, N. (2009). Meanings of 'Ubiquitous Learning'. En Cope, B. y Kalantzis, M. (Eds.), Ubiquitous Learning. Exploring the anywhere/ anytime possibilities for learning in the age of digital media. Illinois: University of Illinois Press.

Fourez, G. (1997). Scientific and technological literacy as social practice. Social Studies of Science, (27), 907-936.

Gray, W. S. (1956). The Teaching of Reading and Writing. Illinois: UNESCO-Scott, Foresman and Company.

Hampson, P. J.; Morris, P. E. (1996). Understanding Cognition. Oxford: Blackwell. Harris, T. L. \& Hodges, R. E. (1995). The Literacy dictionary: The vocabulary of reading and writing. Newark, DE: International Reading Association.

Hurd, P. D. (1958). Science Literacy: Its meaning for American Schools. Educational Leadership 16(1), 13-16.

Kellner, D. (2004). Technological Transformation, Multiple Literacies, and the Revisioning of Education. E-learning, 1(1).

Kahn, R.; Kellner, D. (2006). Reconstructing Technoliteracy: A Multiple Literacies Approach. In Denkers, J. Defining Technological Literacy: Towards an Epistemological Framework. Palgrave.

Kolb, D. A. (1984). Experiential Learning. Experience as a source of learning and development. Englewood Cliffs, NJ: Prentice-Hall.

McCurdy, R.C. (1958). Towards a population literatate in science. The Science Teacher, (25), 366-368.

New London Group (1996). A pedagogy of multiliteracies: Designing social futures. Harvard Educational Review, 66(1), 60-92.

Mezirow, J. (1997). Transformative Learning: Theory to Practice. New directions for adult and continuing education, (74 Summer): http://dx.doi.org/10.1002/ace.7401 OCDE (1995). Statistiques Canada. Paris: OCDE.

Perkins, D. N.; Salomon, G. (1989). Are Cognitive Skills Context-Bound? Educational Researcher, 18(1, Jan.-Feb), 16-25. American Educational Research Association Stable.

Snyder, I. (2001). A new communication order: Researching literacy practices in the network society. Language and Education, 15(2), 117-131.

Sullivan, W.; Rosin, M. (2008). A life of the mind for practice: Bridging liberal and professional education.Change, (Mar./Apr.), 44-47.

Tyner, K.; Gutiérrez Martín, A.; Torrego González, A. (2015). "Multialfabetización" sin muros en la era de la convergencia. La competencia digital y "la cultura del hacer" como revulsivos para una educación continua. Profesorado. Revista de Currículum y Formación de Profesorado [en línea], 19(mayo-agosto). Disponible en: http://www.redalyc.org/articulo.oa?id=56741181004 (consultada el 10/05/2017). Zhou, K. (2016). Non-cognitive Skills: Definitions, measurement and malleability. Global Education Monitoring Report. UNESCO.

El desarrollo de las multialfabetizaciones en... I Camilloni, A. I 